\title{
The best, the worst and the semi-strong: optimal values in interval linear programming
}

\author{
Elif Garajová ${ }^{1, *}$, Milan Hladík ${ }^{1}$ and Miroslav Rada ${ }^{2}$ \\ ${ }^{1}$ Faculty of Mathematics and Physics, Charles University \\ Malostranské nám. 25, 11800 Prague, Czech Republic \\ E-mail: 〈\{elif, hladik\}@kam.mff.cuni.cz〉 \\ ${ }^{2}$ Faculty of Finance and Accounting, University of Economics, Prague \\ nám. W. Churchilla 4, 13067 Prague, Czech Republic \\ E-mail: 〈miroslav.rada@vse.cz〉
}

\begin{abstract}
Interval programming provides one of the modern approaches to modeling optimization problems under uncertainty. Traditionally, the best and the worst optimal values determining the optimal value range are considered as the main solution concept for interval programs. In this paper, we present the concept of semi-strong values as a generalization of the best and the worst optimal values. Semi-strong values extend the recently introduced notion of semi-strong optimal solutions, allowing the model to cover a wider range of applications. We propose conditions for testing values that are strong with respect to the objective vector, right-hand-side vector or the constraint matrix for interval linear programs in the general form.
\end{abstract}

Keywords: linear programming, interval data, optimal value, semi-strong value

Received: September 30, 2018; accepted: October 8, 2019; available online: December 13, 2019

DOI: $10.17535 /$ crorr.2019.0018

\section{Introduction}

Throughout the last years, several different approaches for incorporating uncertainty and inexactness of real-world data into mathematical models have emerged. Interval programming $[6,19]$ provides a tool for representing problems, in which the input data can perturb independently within the given lower and upper bounds. The interval approach has been utilized in various applications, such as portfolio selection [11], transportation problems [1] or input-output models [10].

One of the main questions posed in interval linear programming is the problem of computing or approximating the optimal value range, i.e. finding the best and the worst optimal value over all possible scenarios. Methods for exact calculation of these bounds are available [5, 15, 19], however, it is a computationally challenging problem, in general.

Other questions include properties of classical linear programming studied in the weak or strong sense. In other words, we are interested whether a given property holds for some or for all scenarios of the interval program, e.g. whether a given solution is feasible or optimal for some realization of the input data. However, besides the traditional solution concept of weak feasibility used in interval linear systems, other concepts that were more suited towards particular applications have been proposed. These concepts include tolerance solutions, control solutions [20] or AE solutions [8], which were later unified and extended under the notion

${ }^{*}$ Corresponding author. 
of generalized feasibility $[12,22]$. Recently, a similar concept was also introduced to interval optimization in the form of semi-strong optimality $[9,13,14]$, which serves as a generalization of weak and strong optimality.

In this paper, we present a notion of generalized optimal values in the context of semi-strong optimality. This approach allows to represent two different types of uncertain input data and consider optimal values that are strong with respect to only some of the coefficients, thus providing a natural extension of the best and the worst optimal values. The paper is organized as follows: In Section 2, we review the necessary concepts of interval linear programming. Section 3 addresses the problem of computing the optimal value range and introduces the related notions of weak and strong values. Section 4 presents a generalization to semi-strong values and discusses the corresponding testing conditions. In Section 5, we summarize the results and propose some ideas for further research.

\section{Interval linear programming}

Let us first introduce the essential definitions and notation used in interval linear programming. For a vector $p \in \mathbb{R}^{n}$, we denote by $T_{p}$ the corresponding diagonal matrix with the vector $p$ on the diagonal. Given two real matrices $\underline{A}, \bar{A} \in \mathbb{R}^{m \times n}$ with $\underline{A} \leq \bar{A}$, we define an interval matrix as the set

$$
\boldsymbol{A}=[\underline{A}, \bar{A}]=\left\{A \in \mathbb{R}^{m \times n}: \underline{A} \leq A \leq \bar{A}\right\} .
$$

The center and the radius of an interval matrix $\boldsymbol{A}$ are defined as $A_{c}=\frac{1}{2}(\bar{A}+\underline{A})$ and $A_{\Delta}=$ $\frac{1}{2}(\bar{A}-\underline{A})$, respectively. Hereinafter, we denote interval objects by bold letters. The symbol $\mathbb{R}$ stands for the set of all real intervals.

Given interval matrices $\boldsymbol{A} \in \mathbb{R}^{k \times m}, \boldsymbol{B} \in \mathbb{R}^{k \times n}, \boldsymbol{C} \in \mathbb{R}^{l \times m}, \boldsymbol{D} \in \mathbb{R}^{l \times n}$, right-hand-side vectors $\boldsymbol{b} \in \mathbb{R}^{k}, \boldsymbol{d} \in \mathbb{R}^{l}$ and objective vectors $\boldsymbol{a} \in \mathbb{R}^{m}, \boldsymbol{c} \in \mathbb{R}^{n}$, we define the corresponding interval linear program (ILP) in the general form as a family of linear programs

$$
\begin{array}{ll}
\text { minimize } & a^{T} x+c^{T} y \\
\text { subject to } & A x+B y=b, \\
& C x+D y \leq d, \\
& x \geq 0,
\end{array}
$$

with $A \in \boldsymbol{A}, B \in \boldsymbol{B}, C \in \boldsymbol{C}, D \in \boldsymbol{D}$ and $a \in \boldsymbol{a}, b \in \boldsymbol{b}, c \in \boldsymbol{c}, d \in \boldsymbol{d}$. A particular linear program in the family is called a scenario. For the sake of brevity, we also write an interval linear program as a linear program with interval coefficients, i.e.

$$
\begin{array}{ll}
\operatorname{minimize} & \boldsymbol{a}^{T} x+\boldsymbol{c}^{T} y \\
\text { subject to } & \boldsymbol{A} x+\boldsymbol{B} y=\boldsymbol{b}, \\
& \boldsymbol{C} x+\boldsymbol{D} y \leq \boldsymbol{d}, \\
& x \geq 0 .
\end{array}
$$

While the distinction between equations and inequalities or free and non-negative variables in program (1) may seem unnecessary, note that the standard equivalent transformations known from classical linear programming are not applicable in the interval case due to the so-called dependency problem (see also [3]). By transforming an interval constraint $\boldsymbol{a}^{T} x=b$ into two inequalities $\boldsymbol{a}^{T} x \leq b$ and $\boldsymbol{a}^{T} x \geq b$, we introduce new scenarios into the system that were not present in the original constraint. This is caused by the loss of dependency between the two occurrences of the interval coefficient $\boldsymbol{a}$ in the transformed system: while the original constraint represents the set $\left\{a^{T} x=b: a \in \boldsymbol{a}\right\}$, the latter system represents the larger set $\left\{a_{1}^{T} x \leq b, a_{2}^{T} x \geq b: a_{1}, a_{2} \in \boldsymbol{a}\right\}$.

A natural way of defining an interval counterpart to various properties of linear programs is to study whether the property holds for some scenario (we say it is a weak property) or for each 
scenario (a strong property) of the interval program. For example, a vector $x \in \mathbb{R}^{n}$ is a weakly feasible solution to the interval linear program (2), if it is feasible for some scenario (1) of the program. Furthermore, we say that an ILP itself is weakly feasible, if there exists a feasible scenario. A solution or a program is strongly feasible, if the feasibility property holds for each scenario. For notational simplicity, throughout the text we refer to the data of the constraints of ILP (2) shortly by $\boldsymbol{S}=(\boldsymbol{A}, \boldsymbol{B}, \boldsymbol{C}, \boldsymbol{D}, \boldsymbol{b}, \boldsymbol{d})$ and denote the set of all weakly feasible solutions by $\mathcal{M}(\boldsymbol{S})$.

A condition for testing weak feasibility of an interval linear system of equations (and a description of the weakly feasible set) was given by Oettli and Prager [16], and was later extended to inequality systems by Gerlach [4]. Building on these results, a characterization of weak feasibility for interval linear systems in general form was derived by Hladík [7].

Theorem 1 (see $[4,7,16]$ ). The set of weakly feasible solutions to an interval linear system

$$
\boldsymbol{A} x+\boldsymbol{B} y=\boldsymbol{b}, \boldsymbol{C} x+\boldsymbol{D} y \leq \boldsymbol{d}, x \geq 0
$$

is described by the non-linear system

$$
\begin{aligned}
\underline{A} x+B_{c} y & \leq B_{\Delta}|y|+\bar{b}, \\
-\bar{A} x-B_{c} y & \leq B_{\Delta}|y|-\underline{b}, \\
\underline{C} x+D_{c} y & \leq D_{\Delta}|y|+\bar{d}, \quad x \geq 0 .
\end{aligned}
$$

If all variables of the system are restricted to a single orthant, the characterization given in Theorem 1 reduces to a linear system. Therefore, weak feasibility of a general interval linear system can also be tested by using an orthant decomposition and solving an exponential number of linear systems.

Corollary 1 (see $[4,7,16])$. An interval linear system

$$
\boldsymbol{A} x+\boldsymbol{B} y=\boldsymbol{b}, \boldsymbol{C} x+\boldsymbol{D} y \leq \boldsymbol{d}, x \geq 0
$$

is weakly feasible if and only if the linear system

$$
\begin{aligned}
\underline{A} x+\left(B_{c}-B_{\Delta} T_{p}\right) y & \leq \bar{b}, \\
-\bar{A} x-\left(B_{c}+B_{\Delta} T_{p}\right) y & \leq-\underline{b}, \\
\underline{C} x+\left(D_{c}-D_{\Delta} T_{p}\right) y & \leq \bar{d}, \quad x \geq 0
\end{aligned}
$$

if feasible for some $p \in\{ \pm 1\}^{n}$.

A similar characterization was also derived for testing strong feasibility of an interval system based on the results of Rohn and Kreslová [18, 21].

Theorem 2 (see $[7,18,21])$. An interval linear system

$$
\boldsymbol{A} x+\boldsymbol{B} y=\boldsymbol{b}, \boldsymbol{C} x+\boldsymbol{D} y \leq \boldsymbol{d}, x \geq 0
$$

is strongly feasible if and only if the linear system

$$
\begin{aligned}
\left(A_{c}+T_{p} A_{\Delta}\right) x+\left(B_{c}+T_{p} B_{\Delta}\right) y^{1}-\left(B_{c}-T_{p} B_{\Delta}\right) y^{2} & =b_{c}-T_{p} b_{\Delta} \\
\bar{C} x+\bar{D} y^{1}-\underline{D} y^{2} & \leq \underline{d} \\
x, y^{1}, y^{2} & \geq 0
\end{aligned}
$$

is feasible for each $p \in\{ \pm 1\}^{k}$. 
For the case of strong feasibility, the testing system for inequality constraints is linear. However, for equation-constrained interval systems, the characterization requires an exponential number of linear systems even with non-negative variables.

Note that even if a strongly feasible solution is not likely to exist for a system of interval equations, in general, the system itself can still be strongly feasible. For inequality-constrained systems, the two notions coincide and the strongly feasible solutions can be characterized by Corollary 2.

Corollary 2 (see [20]). A solution $y \in \mathbb{R}^{n}$ is strongly feasible for the system $\boldsymbol{D} y \leq \boldsymbol{d}$ if and only if $y=y^{1}-y^{2}$ for some $y^{1}, y^{2} \in \mathbb{R}^{n}$ satisfying

$$
\bar{D} y^{1}-\underline{D} y^{2} \leq \underline{d}, y^{1} \geq 0, y^{2} \geq 0 .
$$

\section{Optimal value range}

One of the basic questions studied in interval optimization is the problem of computing the best and the worst optimal value over all scenarios, often referred to as the optimal value range. For a classical minimization linear program (1) given by constraint data $S=(A, B, C, D, b, d)$ and an objective vector $(a, c)$, we define the optimal value of the program as

$$
f(S, a, c)=\inf \left\{a^{T} x+c^{T} y:(x, y) \in \mathcal{M}(S)\right\} .
$$

For the optimal value of a linear program, one of the three cases occurs:

- $f(S, a, c)=-\infty$ if the program is unbounded,

- $f(S, a, c)=\infty$ if the program is infeasible,

- $f(S, a, c)=a^{T} x^{*}+c^{T} y^{*}$ if there is an optimal solution $\left(x^{*}, y^{*}\right)$.

Analogously, for an interval linear program (2), the bounds of the optimal value range $[f, \bar{f}]$ enclosing all optimal values (also known as the best and the worst optimal value, respectively) are defined as

$$
\begin{aligned}
& \underline{f}=\inf \{f(S, a, c): S \in \boldsymbol{S}, a \in \boldsymbol{a}, c \in \boldsymbol{c}\}, \\
& \bar{f}=\sup \{f(S, a, c): S \in \boldsymbol{S}, a \in \boldsymbol{a}, c \in \boldsymbol{c}\} .
\end{aligned}
$$

Algorithms for computing the exact bounds $f, \bar{f}$ were proposed for different forms of interval linear programs [15, 19], as well as a unified approach for programs in the general form [2, 5].

Let us now reformulate the problem of computing the optimal value range in the context of weak and strong properties of an ILP. We can say that a value $r$ is a weak value, if there is a scenario of the program with $f(S, a, c) \leq r$. In other words, the value $r$ (or a better one) can be achieved by at least one scenario. Analogously, we can define $r$ as a strong value, if we have $f(S, a, c) \leq r$ for each scenario. Then, the best and the worst optimal values $\underline{f}, \bar{f}$ can be viewed as the best of all weak or strong values, respectively.

Consider an interval linear program in the form (2) and let a value $r \in \mathbb{R}$ be given. Theorems 3 and 4 restate the problem of testing whether the value $r$ is a weak or a strong value in terms of weak and strong feasibility of an interval linear system.

Theorem 3. There exists a scenario $S \in \boldsymbol{S}, a \in \boldsymbol{a}, c \in \boldsymbol{c}$ with $f(S, a, c) \leq r$ if and only if the interval linear system

$$
\boldsymbol{a}^{T} x+\boldsymbol{c}^{T} y \leq r, \boldsymbol{A} x+\boldsymbol{B} y=\boldsymbol{b}, \boldsymbol{C} x+\boldsymbol{D} y \leq \boldsymbol{d}, x \geq 0
$$

is weakly feasible. 
Theorem 4. We have $f(S, a, c) \leq r$ for each $S \in \boldsymbol{S}, a \in \boldsymbol{a}, c \in \boldsymbol{c}$ if and only if the interval linear system

$$
\boldsymbol{a}^{T} x+\boldsymbol{c}^{T} y \leq r, \boldsymbol{A} x+\boldsymbol{B} y=\boldsymbol{b}, \boldsymbol{C} x+\boldsymbol{D} y \leq \boldsymbol{d}, x \geq 0
$$

is strongly feasible.

\section{Semi-strong values}

The definition of weak and strong values introduced in Section 3 leads to a natural generalization of the extremal (best and worst) optimal values, inspired by the concepts of generalized feasibility [22] and semi-strong optimality [13]. Alternative quantifier-based solution concepts for interval programs have been proposed as special cases of generalized feasibility, such as tolerance solutions, control solutions or algebraic solutions (see [20] and references therein), to be used for various practical applications, such as input-output planning [17] and control system design [22].

The concept of weak or strong solutions assumes that the coefficients can only be affected by the same type of uncertainty or inexactness. However, in many applications, some of the coefficients can be controlled by the decision-maker (within a prescribed tolerance), while other suffer from external uncertainty and cannot be influenced. To reflect such distinction in the model, it may be beneficial to consider optimal values that are "strong" with respect to some of the coefficients, but can still be "weak" with respect to others.

Let $\mathcal{U} \subseteq\{\boldsymbol{A}, \boldsymbol{B}, \boldsymbol{C}, \boldsymbol{D}, \boldsymbol{a}, \boldsymbol{b}, \boldsymbol{c}, \boldsymbol{d}\}$ be a set of interval matrices and vectors in the program. Further, define the interval matrices $\boldsymbol{A}^{\forall}, \ldots, \boldsymbol{D}^{\forall}, \boldsymbol{A}^{\exists}, \ldots, \boldsymbol{D}^{\exists}$ and interval vectors $\boldsymbol{a}^{\forall}, \ldots, \boldsymbol{d}^{\forall}$, $\boldsymbol{a}^{\exists}, \ldots, \boldsymbol{d}^{\exists}$ as follows:

$$
\boldsymbol{I}^{\forall}=\left\{\begin{array}{ll}
\boldsymbol{I} & \text { if } \boldsymbol{I} \in \mathcal{U}, \\
0 & \text { if } \boldsymbol{I} \notin \mathcal{U},
\end{array} \quad \boldsymbol{I}^{\exists}= \begin{cases}0 & \text { if } \boldsymbol{I} \in \mathcal{U}, \\
\boldsymbol{I} & \text { if } \boldsymbol{I} \notin \mathcal{U},\end{cases}\right.
$$

for each $\boldsymbol{I} \in\{\boldsymbol{A}, \boldsymbol{B}, \boldsymbol{C}, \boldsymbol{D}, \boldsymbol{a}, \boldsymbol{b}, \boldsymbol{c}, \boldsymbol{d}\}$. We say that a given value $r$ is $\mathcal{U}$-strong, if

$$
\begin{aligned}
& \forall A^{\forall} \in \boldsymbol{A}^{\forall}, \ldots, D^{\forall} \in \boldsymbol{D}^{\forall}, a^{\forall} \in \boldsymbol{a}^{\forall}, \ldots, d^{\forall} \in \boldsymbol{d}^{\forall}, \\
& \exists A^{\exists} \in \boldsymbol{A}^{\exists}, \ldots, D^{\exists} \in \boldsymbol{D}^{\exists}, a^{\exists} \in \boldsymbol{a}^{\exists}, \ldots, d^{\exists} \in \boldsymbol{d}^{\exists}: f(S, a, c) \leq r,
\end{aligned}
$$

where $S=\left(A^{\forall}+A^{\exists}, B^{\forall}+B^{\exists}, C^{\forall}+C^{\exists}, D^{\forall}+D^{\exists}, b^{\forall}+b^{\exists}, d^{\forall}+d^{\exists}\right), a=a^{\forall}+a^{\exists}$ and $c=c^{\forall}+c^{\exists}$. We also refer to such values collectively as semi-strong values.

For example, we say that a given value $r$ is $\{\boldsymbol{A}, \boldsymbol{B}, \boldsymbol{C}, \boldsymbol{D}\}$-strong, if for each $A \in \boldsymbol{A}, B \in \boldsymbol{B}$, $C \in \boldsymbol{C}, D \in \boldsymbol{D}$ there exist $a \in \boldsymbol{a}, b \in \boldsymbol{b}, c \in \boldsymbol{c}, d \in \boldsymbol{d}$ such that $f(S, a, c) \leq r$ for $S=$ $(A, B, C, D, b, d)$. Similarly, a strong value (as defined in Section 3 ) is an $\{\boldsymbol{A}, \boldsymbol{B}, \boldsymbol{C}, \boldsymbol{D}, \boldsymbol{a}, \boldsymbol{b}, \boldsymbol{c}, \boldsymbol{d}\}$ strong value in this sense and a weak value is an $\emptyset$-strong value. In this section, we examine values that are strong with respect to the objective vector, right-hand side or the constraint matrix.

Theorem 5. A value $r \in \mathbb{R}$ is $\{\boldsymbol{a}, \boldsymbol{c}\}$-strong if and only if the interval system

$$
\boldsymbol{a}^{T} x+\boldsymbol{c}^{T} y \leq r,(x, y) \in \mathcal{M}(\boldsymbol{S})
$$

is strongly feasible.

Proof. Assume that for each $a \in \boldsymbol{a}, c \in \boldsymbol{c}$ there exists a scenario $S \in \boldsymbol{S}$ satisfying $f(S, a, c) \leq r$. Then $S$ has a feasible solution $\left(x^{*}, y^{*}\right)$ with $a^{T} x^{*}+c^{T} y^{*} \leq r$, and thus ILP (3) is strongly feasible. On the other hand, strong feasibility of the interval system ensures that for each objective vector $a \in \boldsymbol{a}, c \in \boldsymbol{c}$ we have a weakly feasible solution $\left(x^{*}, y^{*}\right)$ and a corresponding scenario $S$, which satisfy $r \geq a^{T} x^{*}+c^{T} y^{*} \geq f(S, a, c)$. 
Recall that we can describe the weakly feasible solution set $\mathcal{M}(\boldsymbol{S})$ of an interval linear system by the non-linear system presented in Theorem 1. Furthermore, we can also apply the conditions for testing strong feasibility stated in Theorem 2 .

Corollary 3. A value $r \in \mathbb{R}$ is $\{\boldsymbol{a}, \boldsymbol{c}\}$-strong if and only if the system

$$
\begin{aligned}
\bar{a}^{T} x+\bar{c}^{T} y^{1}-\underline{c}^{T} y^{2} & \leq r, \quad y=y^{1}-y^{2}, \\
\underline{A} x+\left(B_{c}-B_{\Delta} T_{p}\right) y & \leq \bar{b}, \\
-\bar{A} x-\left(B_{c}+B_{\Delta} T_{p}\right) y & \leq-\underline{b}, \\
\underline{C} x+\left(D_{c}-D_{\Delta} T_{p}\right) y & \leq \bar{d}, \quad x, y^{1}, y^{2} \geq 0, T_{p} y \geq 0
\end{aligned}
$$

is feasible for some $p \in\{ \pm 1\}^{n}$.

Proof. First, we can write out the weakly feasible set $\mathcal{M}(\boldsymbol{S})$ to obtain the system

$$
\begin{aligned}
\boldsymbol{a}^{T} x+\boldsymbol{c}^{T} y & \leq r, \\
\underline{A} x+B_{c} y-B_{\Delta}|y| & \leq \bar{b}, \\
-\bar{A} x-B_{c} y-B_{\Delta}|y| & \leq-\underline{b}, \\
\underline{C} x+D_{c} y-D_{\Delta}|y| & \leq \bar{d}, \quad x \geq 0 .
\end{aligned}
$$

Assume now that the non-linear system (5) is strongly feasible and consider all quadruples $(a, c, x, y)$ satisfying the constraints. Let $\left(a^{*}, c^{*}, x^{*}, y^{*}\right)$ be a feasible solution maximizing the value $a^{T} x+c^{T} y$. Then $\left(x^{*}, y^{*}\right)$ is feasible for system (5) for each $a \in \boldsymbol{a}, c \in \boldsymbol{c}$, since we have

$$
a^{T} x^{*}+c^{T} y^{*} \leq\left(a^{*}\right)^{T} x^{*}+\left(c^{*}\right)^{T} y^{*} \leq r
$$

and the pair satisfies the absolute-value constraints. Therefore, $\left(x^{*}, y^{*}\right)$ is also a strongly feasible solution of $\boldsymbol{a}^{T} x+\boldsymbol{c}^{T} y \leq r$ and by the characterization of strong solutions (see Corollary 2) and orthant decomposition, it satisfies system (4).

Conversely, if system (4) is feasible for some $p \in\{ \pm 1\}^{n}$, then there exists a strongly feasible solution of $\boldsymbol{a}^{T} x+\boldsymbol{c}^{T} y \leq r$ satisfying the additional constraints. Thus, system (5) is strongly feasible.

We can formulate a similar condition for testing semi-strong values with respect to righthand sides or constraint matrices, as shown in Theorems 6 and 7 .

Theorem 6. A value $r \in \mathbb{R}$ is $\{\boldsymbol{b}, \boldsymbol{d}\}$-strong if and only if the system

$$
\begin{aligned}
\underline{a}^{T} x+c_{c}^{T} y-c_{\Delta}^{T}|y| & \leq r, \\
\underline{A} x+B_{c} y-B_{\Delta}|y| & \leq z, \\
-\bar{A} x-B_{c} y-B_{\Delta}|y| & \leq-z, \\
\underline{C} x+D_{c} y-D_{\Delta}|y| & \leq \underline{d}, \quad x \geq 0, z=\boldsymbol{b}
\end{aligned}
$$

is strongly feasible.

Proof. For fixed $b \in \boldsymbol{b}, d \in \boldsymbol{d}$, there exist the corresponding constraint matrices and objective vectors if and only if the weakly feasible set

$$
\begin{aligned}
\underline{a}^{T} x+c_{c}^{T} y-c_{\Delta}^{T}|y| & \leq r, \\
\underline{A} x+B_{c} y-B_{\Delta}|y| & \leq b, \\
-\bar{A} x-B_{c} y-B_{\Delta}|y| & \leq-b, \\
\underline{C} x+D_{c} y-D_{\Delta}|y| & \leq d, \quad x \geq 0
\end{aligned}
$$

is non-empty. Since the condition should hold for each $b$ and $d$ in their respective intervals, we can set $d=\underline{d}$ as the worst-case scenario and require strong feasibility with respect to $\boldsymbol{b}$. 
Theorem 7. A value $r \in \mathbb{R}$ is $\{\boldsymbol{A}, \boldsymbol{B}, \boldsymbol{C}, \boldsymbol{D}\}$-strong if and only if the system

$$
\begin{aligned}
\underline{a}^{T} x+c_{c}^{T} y-c_{\Delta}^{T}|y| & \leq r, \\
\boldsymbol{A} x+\boldsymbol{B} y & =z, \quad \underline{b} \leq z \leq \bar{b}, \\
\boldsymbol{C} x+\boldsymbol{D} y & \leq \bar{d}, \quad x \geq 0
\end{aligned}
$$

is strongly feasible.

Proof. Again, we can replace the existentially quantified vectors by using the characterization of weak feasibility. Namely, we can describe the weakly feasible set of $\boldsymbol{a}^{T} x+\boldsymbol{c}^{T} y \leq r$ by the constraint $\underline{a}^{T} x+c_{c}^{T} y-c_{\Delta}^{T}|y| \leq r$, substitute the interval vector $\boldsymbol{b}$ by a vector of variables bound to the interval, and the interval $\boldsymbol{d}$ by the best value $\bar{d}$. Strong feasibility of the system ensures that the condition holds for each of the constraint matrices.

Let us now formulate conditions for testing values that are strong with respect to two of the three considered parts of an interval linear program. The proofs of the following theorems are analogous to the previous results.

Theorem 8. A value $r \in \mathbb{R}$ is $\{\boldsymbol{A}, \boldsymbol{B}, \boldsymbol{C}, \boldsymbol{D}, \boldsymbol{a}, \boldsymbol{c}\}$-strong if and only if the interval linear system

$$
\begin{aligned}
& \boldsymbol{a}^{T} x+\boldsymbol{c}^{T} y \leq r, \\
& \underline{b} \leq \boldsymbol{A} x+\boldsymbol{B} y \leq \bar{b}, \boldsymbol{C} x+\boldsymbol{D} y \leq \bar{d}, x \geq 0
\end{aligned}
$$

is strongly feasible.

Theorem 9. A value $r \in \mathbb{R}$ is $\{\boldsymbol{A}, \boldsymbol{B}, \boldsymbol{C}, \boldsymbol{D}, \boldsymbol{b}, \boldsymbol{d}\}$-strong if and only if the system

$$
\begin{aligned}
& \underline{a}^{T} x+c_{c}^{T} y-c_{\Delta}^{T}|y| \leq r, \\
& \boldsymbol{A} x+\boldsymbol{B} y=\boldsymbol{b}, \boldsymbol{C} x+\boldsymbol{D} y \leq \boldsymbol{d}, x \geq 0
\end{aligned}
$$

is strongly feasible.

Theorem 10. A value $r \in \mathbb{R}$ is $\{\boldsymbol{a}, \boldsymbol{b}, \boldsymbol{c}, \boldsymbol{d}\}$-strong if and only if the system

$$
\begin{aligned}
\bar{a}^{T} x+c^{T} y & \leq r, \\
\underline{A} x+B_{c} y-B_{\Delta}|y| & \leq z, \\
-\bar{A} x-B_{c} y-B_{\Delta}|y| & \leq-z, \\
\underline{C} x+D_{c} y-D_{\Delta}|y| & \leq \underline{d}, \quad x \geq 0, z=\boldsymbol{b}
\end{aligned}
$$

is strongly feasible.

The resulting conditions for testing semi-strong values can also be described by means of so-called AE feasibility of an interval system [8]. Note that the concept of semi-strong values can be generalized even further by considering a finer partition of the coefficients or choosing a different order of the quantifiers.

\section{Conclusion}

We introduced the concept of semi-strong values as a generalization of the optimal value range in interval linear programming. Semi-strong values provide a natural extension of the best and the worst optimal values and form a complement to the notions of generalized feasibility and semi-strong optimal solutions. By weakening the definition of optimal values and considering values that are strong only with respect to some of the uncertain input data, we allow the model to reflect different controllability over various parts of the program. We derived testing conditions for several types of semi-strong values related to AE feasibility of interval systems. Since the best semi-strong values may be of interest, these conditions can also serve as a basis for designing a method to compute such generalized optimal values. 


\section{Acknowledgement}

The first two authors were supported by the Czech Science Foundation under Grant P403-1804735S and by the Charles University, project GA UK No. 156317. The third author was supported by the Czech Science Foundation under Grant P403-17-13086S.

\section{References}

[1] Cerulli, R., D'Ambrosio, C., and Gentili, M. (2017). Best and Worst Values of the Optimal Cost of the Interval Transportation Problem. In Optimization and Decision Science: Methodologies and Applications, Springer Proceedings in Mathematics \& Statistics, 367374. doi:10.1007/978-3-319-67308-0_37

[2] Chinneck, J. W., and Ramadan, K. (2000). Linear Programming with Interval Coeffcients. Journal of the Operational Research Society, 51, 2, 209-220. doi:10.1057/palgrave.jors.2600891

[3] Garajová, E., Hladík, M., and Rada, M. (2018). Interval Linear Programming under Transformations: Optimal Solutions and Optimal Value Range. Central European Journal of Operations Research, 27(3), 601-614. doi:10.1007/s10100-018-0580-5

[4] Gerlach, W. (1981). Zur Lösung Linearer Ungleichungssysteme Bei Störung Der Rechten Seite Und Der Koeffzientenmatrix. Optimization, 12, 41-43. doi:10.1080/02331938108842705

[5] Hladík, M. (2009). Optimal Value Range in Interval Linear Programming. Fuzzy Optimization and Decision Making, 8, 3, 283-294. doi:10.1007/s10700-009-9060-7

[6] Hladík, M. (2012). Interval Linear Programming: A Survey. In Mann, Z. A. (Ed.), Linear Programming - New Frontiers in Theory and Applications, chapter 2, (pp. 85-120). New York: Nova Science Publishers

[7] Hladík, M. (2013). Weak and Strong Solvability of Interval Linear Systems of Equations and Inequalities. Linear Algebra and its Applications, 438, 11, 4156-4165. doi:10.1016/j.laa.2013.02.012

[8] Hladík, M. (2015). AE Solutions and AE Solvability to General Interval Linear Systems. Linear Algebra and its Applications, 465, 221-238. doi:10.1016/j.laa.2014.09.030

[9] Hladík, M. (2017). On Strong Optimality of Interval Linear Programming. Optimization Letters, 11, 7, 1459-1468. doi:10.1007/s11590-016-1088-3

[10] Jerrell, M. E. (1997). Interval Arithmetic for Input-Output Models with Inexact Data. Computational Economics, 10, 1, 89-100. doi:10.1023/A:1017987931447

[11] Lai, K. K., Wang, S. Y., Xu, J. P., Zhu, S. S., and Fang, Y. (2002). A Class of Linear Interval Programming Problems and Its Application to Portfolio Selection. IEEE Transactions on Fuzzy Systems, 10, 6, 698-704. doi:10.1109/TFUZZ.2002.805902

[12] Li, H., Luo, J., and Wang, Q. (2014). Solvability and Feasibility of Interval Linear Equations and Inequalities. Linear Algebra and its Applications, 463, 78-94. doi:10.1016/j.laa.2014.08.027

[13] Li, W., Liu, X., and Li, H. (2015). Generalized Solutions to Interval Linear Programmes and Related Necessary and Suffcient Optimality Conditions. Optimization Methods and Software, 30, 3, 516-530. doi:10.1080/10556788.2014.940948 
[14] Luo, J., Li, W., and Wang, Q. (2014). Checking Strong Optimality of Interval Linear Programming with Inequality Constraints and Nonnegative Constraints. Journal of Computational and Applied Mathematics, 260, 180-190. doi:10.1016/j.cam.2013.09.075

[15] Mráz, F. (1998). Calculating the Exact Bounds of Optimal Values in LP with Interval Coeffcients. Annals of Operations Research, 81, 0, 51-62. doi:10.1023/A:1018985914065

[16] Oettli, W., and Prager, W. (1964). Compatibility of Approximate Solution of Linear Equations with given Error Bounds for Coeffcients and Right-Hand Sides. Numerische Mathematik, 6, 1, 405-409.

[17] Rohn, J. (1978). Input-output planning with inexact data. Freiburger Intervall-Berichte, $9,78,1-16$.

[18] Rohn, J. (1981). Strong Solvability of Interval Linear Programming Problems. Computing, 26, 1, 79-82. doi:10.1007/BF02243426

[19] Rohn, J. (2006). Interval Linear Programming. In Fiedler, M., Nedoma, J., Ramík, J., Rohn, J., and Zimmermann, K. (Eds.), Linear Optimization Problems with Inexact Data, (pp. 79-100). Boston, MA: Springer US.

[20] Rohn, J. (2006). Solvability of Systems of Interval Linear Equations and Inequalities. In Fiedler, M., Nedoma, J., Ramík, J., Rohn, J., and Zimmermann, K. (Eds.), Linear Optimization Problems with Inexact Data, (pp. 35-77). Boston, MA: Springer US.

[21] Rohn, J., and Kreslová, J. (1994). Linear Interval Inequalities. Linear and Multilinear Algebra, 38, 1-2, 79-82. doi:10.1080/03081089508818341

[22] Shary, S. P. (2002). A New Technique in Systems Analysis Under Interval Uncertainty and Ambiguity. Reliable Computing, 8, 5, 321-418. doi:10.1023/A:1020505620702 